\section{Kurz notiert}

\section{Ärzte scheuen Grippeimpfung} - für sich selbst

Im vergangenen Herbst zeigte eine Befragung durch das RKI: $40 \%$ der niedergelassenen Ärzte lassen sich nicht regelmäßig gegen Influenza impfen. Noch schlimmer ist die Lage in den Kliniken. Jüngst war im „Bundesgesundheitsblatt" eine Studie zu lesen, in der die Impfquoten von Mitarbeitern der Hessischen Krankenhausgesellschaft untersucht worden waren (S. Wicker et la. Bundesgesundheitsbl 2012; 55: 932-6). In 80\% der Häuser war höchstens jeder fünfte Mitarbeiter gegen Influenza geimpft. Und in der Hälfte der Kliniken, in denen die Raten der einzelnen Berufsgruppen erfasst waren, lag der Anteil der Geimpften unter den Ärzten noch niedriger als beim Pflegepersonal.

$\mathrm{RB}$ -

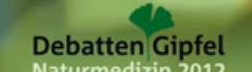

\section{Im Fokus: Naturmedizin}

Beim "Debatten-Gipfel Naturmedizin" am 24. 10. $2012 \mathrm{im}$ Museum für Völkerkunde in Hamburg werden sich Prof. Dr. G. Glaeske, Leiter der Abt. Arzneimittelforschung, Zentrum für Sozialpolitik an der Uni. Bremen und Prof. Dr. M. Habs, Professor für experimentelle Medizin und Geschäftsführer der Dr. Willmar Schwabe GmbH \& Co. KG, unterstützt von weiteren hochkarätigen Disputanten aus Medizin und Pharmakologie ein Streitgespräch liefern. Unter dem Thema "Wenn Wissen auf Leben trifft: Steht der Patient im Mittelpunkt oder im Weg?" geht es um die Frage „Ist die strikte Forderung nach rein evidenzbasierten Arzneimitteln Stufe I bei der Naturmedizin sinnvoll und inwieweit kann oder muss der Patient mit seinen individuellen Bedürfnissen berücksichtigt werden?" Ganz im englischen Debattenstil wertet am Ende das Auditorium die Argumente durch Abstimmung.

Veranstalter: Dr. Willmar Schwabe GmbH \& Co. KG. Medienpartner: Ärzte Zeitung und Springer Medizin. Anmeldung: www.schwabe.de \title{
Zellverjüngung für die Vielfalt
}

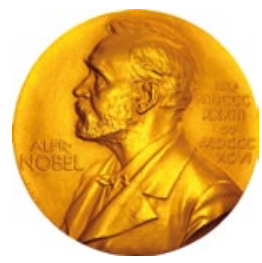

\begin{abstract}
- Der Medizinnobelpreis ging in diesem Jahr zu gleichen Teilen an zwei Wissenschaftler, denen es gelungen ist, bereits ausgereifte Zellen zu reprogrammieren und damit zu verjüngen: an den britischen Professor Sir John B. Gurdon aus Cambridge und an den Japaner Professor Shinya
\end{abstract} Yamanaka aus Kyoto.

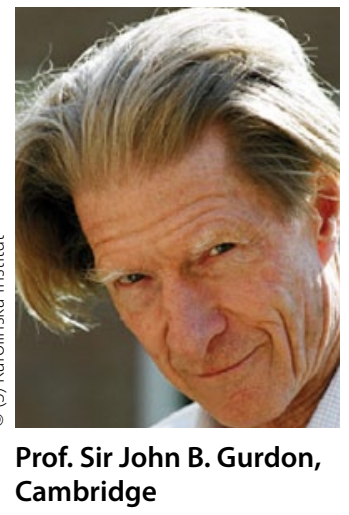
mal werden diese Zellen aber vorrangig in der pharmakologischen Forschung zur Entwicklung neuer Medikamente angewendet werden, wie bei der Pressekonferenz am KarolinskaInstitut in Stockholm betont wurde.

Die grundlegenden Vorarbeiten für die Herstellung von reprogrammierten und damit pluripotenten Stammzellen hat Gurdon bereits in den 1960er-Jahren Vielen, die sich für den Nobelpreis und die Stammzellforschung interessieren, ist das, was Gurdon und Yamanaka entdeckt haben, inzwischen offenbar längst geläufig: Denn auf die Frage, ob sie wussten, dass sich bereits ausgereifte Zellen wieder in unreife Zellen verjüngen lassen, antworteten vor kurzem auf der Internetseite des Nobelpreiskomitees von knapp 8000 Internetusern fast $70 \%$ mit "ja”.

Für den Neuropathologen und Stammzellforscher Professor Oliver Brüstle aus Bonn gelang Yamanaka mit seinem Forschungserfolg 2006 "der große Wurf". Wie Brüstle im Interview mit "Springer Medizin"

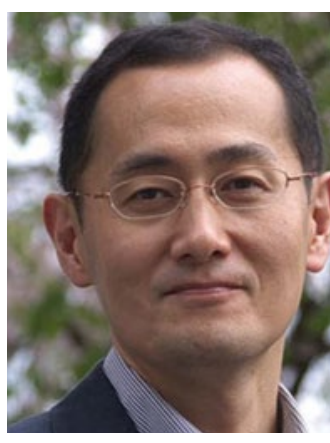

Prof. Shinya Yamanaka, Kyoto sagte, „können wir heute mühelos unbegrenzte Mengen von Gehirn- und Rückenmarkszellen aus einer zwei Millimeter großen Hautprobe oder auch aus Blutzellen gewinnen".

Damit sei es nun möglich, Erbkrankheiten direkt an den betroffenen Nervenzellen zu erforschen und die Wirksamkeit von Arzneien für den einzelnen Patienten zu überprüfen. Eine klinische Anwendung liegt allerdings noch in weiter Ferne. Doch die Herstellung autologer Zellen für den Zellersatz und der Aufbau von Spenderbanken ähnlich jenen für die Knochenmarkstransplantation werden derzeit intensiv erforscht.

Langfristiges Ziel der Forschung mit reprogrammierten Zellen ist es, Gewebe für die regenerative Medizin zu züchten, um defektes Gewebe zu ersetzen. Zunächst ein- geleistet. Er wies nach, dass das Genom einer bereits spezialisierten Zelle noch alle Informationen enthält, die sie für die Verwandlung in die mehr als 200 verschiedenen Zellarten benötigt - und dass sich das fortgeschrittene Entwicklungsstadium tatsächlich umkehren lässt. Gurdon begründete zudem das Klonen. Yamanaka gelang es 2006 als Erstem, zunächst ausdifferenzierte Mauszellen und später dann menschliche Zellen zu reprogrammieren. Diese Zellen werden als induzierte pluripotente Stammzellen bezeichnet und können in der Kulturschale zum Beispiel in Neuronen des zentralen Nervensystems verwandelt werden.

Gurdon, 1933 in Großbritannien geboren, ist Professor für Zellbiologie und forscht heute am Wellcome Trust/Cancer Research UK Gurdon Institute in Cambridge. Yamanaka wurde 1963 in Japan geboren. Er forscht an der Universität in Kyoto sowie am Gladstone-Institut in San Francisco.

Der Nobelpreis für Physiologie oder Medizin war in diesem Jahr mit umgerechnet 930000 Euro (8 Millionen Schwedische Kronen) dotiert - und damit etwa $20 \%$ geringer als bisher. Traditionsgemäß wird der Nobelpreis am 10. Dezember, dem Todestag des Preisstifters Alfred Nobel, überreicht.
Peter Leiner . 